\title{
Hypocretin and brain $\beta$-amyloid peptide interactions in cognitive disorders and narcolepsy
}

\author{
Yves A. Dauvilliers $^{1,2 *}$, Sylvain Lehmann ${ }^{3}$, Isabelle Jaussent ${ }^{2}$ and Audrey Gabelle ${ }^{3,4}$ \\ 'Sleep Unit, National Reference Network for Orphan Diseases (Narcolepsy, Hypersomnia, Kleine-Levin Syndrome), Department of Neurology, Gui de Chauliac \\ Hospital, CHU Montpellier, Montpellier, France \\ 2 INSERM, U1061, Montpellier 1 University, Montpellier, France \\ ${ }^{3}$ Biochimie-Protéomique Clinique - IRMB - CCBHM -Inserm U1040 CHU Montpellier, Montpellier, France \\ ${ }^{4}$ Clinical Research Memory Center Languedoc-Roussillon, Gui de Chauliac Hospital, CHU Montpellier, Montpellier, France
}

\section{Edited by:}

Jean Mariani, Universite Pierre et Marie Curie, France

Reviewed by:

Changiz Geula, Northwestern

University, USA

Leonel E. Rojo, Rutgers University, USA

\section{${ }^{*}$ Correspondence:}

Yves A. Dauvilliers, Sleep Unit, Department of Neurology, Gui de Chauliac Hospital, 80 Avenue Augustin Fliche, 34295 Montpellier Cedex 5, Montpellier, France e-mail:ydauvilliers@yahoo.fr

\begin{abstract}
Objective: To examine relationships between cerebrospinal fluid (CSF) Alzheimer' disease (AD) biomarkers and hypocretin-1 levels in patients with cognitive abnormalities and hypocretin-deficient narcolepsy-cataplexy (NC), estimate diagnostic accuracy, and determine correlations with sleep disturbances.
\end{abstract}

Background: Sleep disturbances are frequent in AD. Interactions between brain $\beta$-amyloid $(A \beta)$ aggregation and a wake-related neurotransmitter hypocretin have been reported in a mouse model of $A D$.

Methods: Ninety-one cognitive patients (37 AD, 16 mild cognitive impairment- $\mathrm{MCl}$ that converts to $A D, 38$ other dementias) and 15 elderly patients with NC were recruited. Patients were diagnosed blind to CSF results. CSF $A \beta_{42}$, total tau, ptau 181 , and hypocretin-1 were measured. Sleep disturbances were assessed with questionnaires in 32 cognitive patients.

Results: Lower CSF $A \beta_{42}$ but higher tau and P-tau levels were found in $A D$ and $\mathrm{MCl}$ compared to other dementias. CSF hypocretin-1 levels were higher in patients with $\mathrm{MCl}$ due to $A D$ compared to other dementias, with a similar tendency for patients with advanced AD. CSF hypocretin-1 was significantly and independently associated with $A D / M C l$ due to $A D$, with an $O R$ of 2.70 after full adjustment, exceeding that for $A \beta_{42}$. $A \beta_{42}$ correlated positively with hypocretin-1 levels in advanced stage AD. No association was found between sleep disturbances and CSF biomarkers. No patients with NC achieved pathological cutoffs for $A \beta_{42}$, with respectively one and four patients with $\mathrm{NC}$ above tau and P-tau cutoffs and no correlations between hypocretin-1 and other biomarkers.

Conclusions: Our results suggest a pathophysiological relationship between $A \beta_{42}$ and hypocretin-1 in the AD process, with higher CSF hypocretin-1 levels in early disease stages. Further longitudinal studies are needed to validate these biomarker interactions and to determine the cause-effect relationship and the role of wake/sleep behavior in amyloid plaque regulation.

Keywords: hypocretin, $\beta$-amyloid, Alzheimer's disease, cognition, sleep, CSF, tau

\section{INTRODUCTION}

Alzheimer's disease $(\mathrm{AD})$ is a neurodegenerative disorder that is expected to become a disastrous worldwide epidemic within 40 years unless treatments to delay or halt the disease are developed. The pathological process is characterized by extracellular aggregation of $\beta$-amyloid peptides $(A \beta)$ produced by amyloid precursor protein (APP) overexpression in the senile plaques and by intra-neuronal tau proteins in particular brain regions (Braak and Braak, 1996; Duyckaerts et al., 2009). However, the mechanisms of this pathological process remain only partly understood. In recent years, there is growing evidence that circadian and sleep disorders may play a causal role in the pathophysiology of AD (Kang et al., 2009; Roh et al., 2012). Sleep disturbances are frequently reported in $\mathrm{AD}$, with both daytime sleepiness and nighttime awakenings (Dauvilliers, 2007; Ju et al., 2013; Spira et al., 2013). Non-specific factors associated with the ageing process and more specific ones, including disrupted sleep and circadian rhythm regulatory systems, are involved in sleep/wake disturbances (Montplaisir et al., 1995; Dauvilliers, 2007; Ju et al., 2013; Slats et al., 2013; Spira et al., 2013). Recently, interactions between the amyloid process and the sleep-wake switching system have been proposed. Brain interstitial fluid $A \beta$ concentration increased during wakefulness and decreased during sleep in an APP transgenic mouse model of AD (Kang et al., 2009). Similar data were found in young healthy subjects, with peak CSF A $\beta$ concentrations in the evening and lower concentrations 
overnight (Bateman et al., 2007; Kang et al., 2009). Further interactions were reported between brain $A \beta$ aggregation and the wake-related neurotransmitter hypocretin/orexin in APP transgenic mice, with increased $A \beta$ levels during hypocretin infusion and decreased levels under the effect of a hypocretin receptor antagonist (Kang et al., 2009). Significantly fewer hypocretin neurons were found in the post-mortem hypothalami of $\mathrm{AD}$ patients (Fronczek et al., 2012). However, several studies have reported a normal range of CSF hypocretin-1 concentration in AD patients (Mignot et al., 2002; Dauvilliers et al., 2003; Baumann et al., 2004; Friedman et al., 2007). One elegant study using an indwelling intrathecal catheter to collect hourly CSF samples in six patients with $\mathrm{AD}$ and six controls reported a correlation between hypocretin-1 and $A \beta_{42}$, with no significant between-group differences in hypocretin-1 circadian rhythm amplitude (Slats et al., 2012). Moreover, lower CSF hypocretin1 levels were found in dementia with Lewy bodies compared to $\mathrm{AD}$ and controls, with no associations between hypocretin1 and $A \beta_{42}$ concentrations (Wennstrom et al., 2012). However, CSF hypocretin- 1 and tau levels were correlated in female nondemented controls (Wennstrom et al., 2012). Thus, the relationships between specific AD biomarkers and hypocretin-1 remain unclear, as well as relationships between sleep/wake states and brain $A \beta$ fluctuations.

Our objective was to investigate the relationships between CSF $A \beta_{42}$, tau, P-tau, and hypocretin-1 in a large population of patients with cognitive abnormalities, including patients at early and advanced stages of $\mathrm{AD}$, patients affected with other dementias, and hypocretin-deficient patients with narcolepsy-cataplexy (NC).

\section{MATERIALS AND METHODS PATIENTS}

One hundred and six unrelated patients (60 males, 46 females; median age 69.3 years; range 32.1-89.5) participated. Patients with cognitive abnormalities comprised a small randomized sample from the Montpellier Cognitive biobank. Over 3000 baseline CSF samples were collected from patients attending the Montpellier Neurological and Clinical Research Memory Center (CMRR) for cognitive and behavioral disorders (official registration No. DC-2008-417). All patients underwent an extensive examination, including physical, neurological, and neuropsychological assessments, laboratory tests, and brain imaging. Diagnoses were performed blind to CSF results and by consensus by a multidisciplinary team of neurologists, geriatricians, and neuropsychologists. We selected 91 patients with cognitive complaints, including 37 with $\mathrm{AD}, 16$ with amnesic mild cognitive impairment (MCI), and 38 with other dementias (DEM). Patients with AD met NINCDS-ADRDA clinical diagnostic criteria (McKhann et al., 1984, 2011). Patients with MCI initially met the usual criteria established by Petersen et al. (1999). All subjects that showed progressive cognitive decline to $\mathrm{AD}$ at a 2-to-7-year follow-up were considered MCI due to AD. The DEM group included frontotemporal lobar degeneration (FTLD) according to consensus criteria (McKhann et al., 2001), dementia with Lewy bodies (LBD) according to McKeith's criteria (McKeith et al., 1996; McKeith, 2006), and corticobasal degeneration according to Boeve et al.'s criteria (Boeve et al., 1999). Dementia severity was assessed using the Mini-Mental State Examination (MMSE) (Folstein et al., 1975).

Elderly patients with typical NC (American Academy of Sleep Medicine, 2005; Dauvilliers et al., 2007) who were followed at the Sleep Unit and with available CSF were included $(n=15 ; 9$ males, 6 females; median age 65.6 years; range 54.0-86.4). Each patient had been clinically diagnosed with NC by a sleep specialist based on at least one night of polysomnographic recording followed by the Multiple Sleep Latency Test (MSLT), HLA-DQB1*0602 typing, and low CSF hypocretin-1 levels $(<110 \mathrm{pg} / \mathrm{mL}$ ) (Mignot et al., 2002; Dauvilliers et al., 2003, 2007). No patients with NC had cognitive abnormalities.

All patients gave their written informed consent to participate in the study. This study was approved by the regional ethics committee of the University of Montpellier Hospital.

\section{CSF SAMPLES AND ASSAYS}

CSF was obtained after a median duration of one month [interquartile range (IQR) 1-3 months] after diagnosis of cognitive abnormalities, in contrast to $\mathrm{NC}$, with more than 30 years of delay between symptom onset and lumbar puncture in this elderly population. CSF was collected in polypropylene tubes under standardized conditions, preferably between 11:00 a.m. and 1:00 p.m. to minimize the influence of diurnal variation in CSF $A \beta_{42}$ levels. CSF samples were transferred within $4 \mathrm{~h}$ after collection and centrifuged at $1000 \mathrm{~g}$ for $10 \mathrm{~min}$ at $4^{\circ} \mathrm{C}$. A small amount of CSF was used for routine analyses, including total cell count, bacteriological exam, and total protein and glucose levels. CSF was aliquoted in polypropylene $1.5 \mathrm{~mL}$ tubes and stored at $-80^{\circ} \mathrm{C}$ until further analysis. CSF $A \beta_{42}$, total tau, and phospho-tau- 181 (P-tau) were measured with Innotest ${ }^{\circledR}$ sandwich ELISA according to manufacturer's procedures (Innogenetics, Ghent, Belgium). The three biomarkers in each CSF sample were analyzed simultaneously. From these measurements, an Innotest ${ }^{\circledR}$ Amyloid Tau Index (IATI) was calculated for each patient. We used validated cutoffs for these biomarkers to clinically discriminate $\mathrm{AD}$ from normal aging and other neurologic disorders (Hulstaert et al., 1999).

Samples were rethawed for CSF hypocretin-1 measurement. CSF hypocretin-1 (orexin-A) was determined in duplicate for all subjects using I radioimmunoassay kits (Phoenix Peptide, Inc.) according to manufacturer's instructions. The detection limit was $10 \mathrm{pg} / \mathrm{mL}$ and intra-assay variability was $<10 \%$. CSF hypocretin1 levels $<110 \mathrm{pg} / \mathrm{mL}$ were considered low, intermediate from 110 to 200 , and normal at $>200$. All values were back-referenced to Stanford reference samples (HHMI Stanford University Center for Narcolepsy, Palo Alto CA) (Mignot et al., 2002). The biological teams analyzed the CSF samples blind to clinical diagnosis.

\section{SLEEP DISTURBANCES}

To assess sleep disturbances, we conducted a short interview and administered a sleep questionnaire in a subgroup of 32 patients ( $n=20 \mathrm{AD} ; n=9 \mathrm{MCI}$ due to AD; $n=3 \mathrm{DEM})$ within 2 years after CSF lumbar puncture. Patients were asked to rate their sleep duration based on bedtime, sleep latency, number of awakenings during the night, duration of awakenings, estimated total 
duration of nighttime sleep hours, and estimated total duration of wake time after sleep onset. The Epworth Sleepiness Scale was used to assess daytime sleepiness. Sleep questionnaires were completed by patients with the help of caregivers when required, especially for patients with AD and DEM.

\section{STATISTICAL ANALYSIS}

The sample is described in percentages for categorical variables and median and range for quantitative variables (age, CSF measurements), with distributions skewed using the Shapiro-Wilk statistic. Chi-square tests or Kruskall-Wallis tests were used to compare categorical and continuous characteristics between the four groups ( $\mathrm{AD}, \mathrm{MCI}$ due to $\mathrm{AD}, \mathrm{DEM}$, and $\mathrm{NC}$ ). Two-group comparisons were performed using the Chi square (categorical variable) and Mann-Whitney Test (continuous variable). When comparisons were statistically significant, two-by-two comparisons were performed using the Bonferroni correction.
Spearman's rank order correlations were applied to determine associations between two continuous variables. In order to determine which CSF measurements were independently associated with $\mathrm{AD}+\mathrm{MCI}$ due to $\mathrm{AD}$, measurements were entered simultaneously into a logistic regression model with potential confounders (e.g., age). Associations were thus quantified with odds ratios (OR) and their 95\% confidence intervals (CI). Significance level was set at $p<0.05$. Statistical analyses were performed using SAS, version 9.2 (SAS Institute, Cary, NC, USA).

\section{RESULTS}

\section{DEMOGRAPHIC, CLINICAL, AND BIOLOGICAL RESULTS BY} DIAGNOSTIC CATEGORY

Results on the demographic, clinical, and biological variables by diagnostic group are reported in Table 1. Significant group differences were observed for age, with patients with $\mathrm{AD}$ being the oldest and patients with NC the youngest. As expected, MMSE

Table 1 | Sociodemographic, clinical, and biological variables for patients with different etiologies of cognitive impairment and patients with narcolepsy-cataplexy.

\begin{tabular}{|c|c|c|c|c|c|c|}
\hline & $\begin{array}{c}\text { AD } \\
N=37\end{array}$ & $\begin{array}{l}\mathrm{MCl} \text { due to } \mathrm{AD} \\
\qquad N=16\end{array}$ & $\begin{array}{c}\text { DEM } \\
N=38\end{array}$ & $\begin{array}{c}N C \\
N=15\end{array}$ & $p$-value & $p$-value ${ }^{a}$ \\
\hline Age (years) & 72.30 [49.80-89.49] & 73.20 [57.39-84.39] & $66.84[32.07-85.15]$ & 65.59 [54.04-86.38] & 0.03 & \\
\hline \multicolumn{7}{|l|}{ Gender } \\
\hline Male & 45.95 & 62.50 & 63.16 & 60.00 & 0.44 & \\
\hline Female & 54.05 & 37.50 & 36.84 & 40.00 & & \\
\hline MMSE & $21.00[2.00-25.00]$ & $26.00[26.00-30.00]$ & $20.50[9.00-30.00]$ & $30.00[30.00-30.00]$ & $<0.0001$ & \\
\hline \multicolumn{7}{|l|}{ MMSE } \\
\hline$<26$ & 100.00 & 0.00 & 80.56 & 0.00 & $<0.0001$ & \\
\hline$\geq 26$ & 0.00 & 100.00 & 19.44 & 100.00 & & \\
\hline \multicolumn{7}{|c|}{ CSF BIOMARKER MEASUREMENT } \\
\hline$A \beta_{42}(p g / m l)$ & 563.00 [232.00-1811.00] & 593.00 [273.00-1505.00] & 694.00 [306.00-1645.00] & 947.00 [566.00-1332.00] & 0.0005 & 0.02 \\
\hline \multicolumn{7}{|l|}{$\mathrm{A} \beta_{42}(\mathrm{pg} / \mathrm{ml})$} \\
\hline$\leq 500$ & 37.84 & 31.25 & 21.05 & 0.00 & NA & NA \\
\hline$>500$ & 62.16 & 68.75 & 78.95 & 100.00 & & \\
\hline Tau (pg/ml) & 579.00 [283.00-2165.00] & 706.50 [343.00-1200.00] & 264.50 [104.00-1200.00] & 215.00 [69.00-633.00] & $<0.0001$ & $<0.0001$ \\
\hline \multicolumn{7}{|l|}{ Tau (pg/ml) } \\
\hline$\geq$ cutoff $^{b}$ & 70.27 & 68.75 & 31.58 & 6.67 & $<0.0001$ & \\
\hline$<$ cutoff & 29.73 & 31.25 & 68.42 & 93.33 & & \\
\hline P-tau (pg/ml) & 79.00 [20.00-215.00] & 106.50 [46.00-215.00] & $41.50[8.00-156.00]$ & 41.00 [19.00-92.00] & $<0.0001$ & $<0.0001$ \\
\hline \multicolumn{7}{|l|}{ P-tau (pg/ml) } \\
\hline$\geq 60$ & 86.49 & 87.50 & 18.42 & 26.67 & $<0.0001$ & $<0.0001$ \\
\hline$<60$ & 13.51 & 12.50 & 81.58 & 73.33 & & \\
\hline IATI index & $0.62[0.00-2.53]$ & 0.60 [0.29-2.33] & $1.28[0.34-3.55]$ & $1.76[1.05-2.28]$ & $<0.0001$ & $<0.0001$ \\
\hline \multicolumn{7}{|l|}{ IATI index } \\
\hline$<1$ & 89.19 & 87.50 & 43.24 & 0.00 & $<0.0001$ & 0.0009 \\
\hline$\geq 1$ & 10.81 & 12.50 & 56.76 & 100.00 & & \\
\hline Hypocretin-1 (pg/ml) & 451.00 [199.00-672.50] & 503.75 [299.00-628.50] & 386.00 [273.00-574.00] & 34.00 [10.00-108.00] & 0.03 & 0.06 \\
\hline \multicolumn{7}{|l|}{ Hypocretin-1 (pg/ml) } \\
\hline$<110$ & 0.00 & 0.00 & 0.00 & 100.00 & NA & NA \\
\hline $110-200$ & 2.70 & 0.00 & 0.00 & 0.00 & & \\
\hline$\geq 200$ & 97.30 & 100.00 & 100.00 & 0.00 & & \\
\hline
\end{tabular}

${ }^{a}$ Adjusted for age.

${ }^{b} 300 \mathrm{pg} / \mathrm{ml}$ (if aged 21-50 years), 450 (if aged 51-70 years), and 500 (if aged > 71 years).

NA, Not applicable. 
differed between groups, with scores for patients with either MCI due to AD or NC above the cutoff of 26. No gender effect was found on any CSF biomarker levels.

As expected, CSF hypocretin-1 levels differed between NC and other diagnostic groups ( $p<0.0001$ for all comparisons), with higher CSF hypocretin-1 levels in MCI due to $\mathrm{AD}, \mathrm{AD}$, and DEM groups compared to NC. Of the patients with cognitive abnormalities, CSF hypocretin-1 levels were higher in MCI due to $\mathrm{AD}$ compared to $\mathrm{DEM}(p=0.02)$, with a similar tendency between $\mathrm{AD}$ and DEM ( $p=0.06$ ) (Figure 1). CSF biomarker levels, including $A \beta_{42}$, tau, P-tau, and IATI, differed significantly between groups regardless of diagnostic category (continuous and categorically validated cutoffs) (Table 1). More precisely, CSF $\mathrm{A} \beta_{42}$ levels differed between the $\mathrm{AD}$ and $\mathrm{NC}(p<0.0006)$ and between the MCI due to AD and NC groups $(p=0.013)$. Significant between-group differences were also found for CSF tau, P-tau, and IATI levels in the $\mathrm{AD}$ vs. DEM, $\mathrm{AD}$ vs. NC, MCI due to $\mathrm{AD}$ vs. DEM, and MCI due to AD vs. NC groups ( $p<0.006$ for all comparisons after Bonferroni correction). No patients with $\mathrm{NC}$ achieved pathological cutoffs for $\mathrm{A} \beta_{42}$ or IATI, with respectively one and four patients with $\mathrm{NC}$ above tau and P-tau cutoffs, all patients without cognitive abnormalities (Table 1). One patient with MCI due to AD had intermediate CSF hypocretin-1 levels, and no patients with either MCI due to AD, $\mathrm{AD}$, or DEM had low hypocretin-1 levels.

\section{COMPARISONS BETWEEN THE AD AND MCI DUE TO AD AND DEM GROUPS}

Because no CSF biomarker levels differed between the AD and $\mathrm{MCI}$ due to $\mathrm{AD}$ groups, and because follow-ups showed that all
MCI developed $\mathrm{AD}$, we decided to pool these two groups into one (AD+MCI-AD). The AD group included patients at early stage (MCI due to $\mathrm{AD}$ ) and advanced stage ( $\mathrm{AD}$ per se) of a similar disease. We further compared sociodemographic, clinical, and biological characteristics of patients with AD+MCI-AD and DEM (Table 2). A between-group age difference was found, with older patients in the AD+MCI-AD group. All CSF biomarker levels differed between groups, with lower $\mathrm{A} \beta_{42}$ and IATI and higher tau and P-tau levels in the AD+MCI-AD group. CSF hypocretin1 levels also differed between groups, with higher levels in the $\mathrm{AD}+\mathrm{MCI}-\mathrm{AD}$ group (Table 2).

Two multivariate logistic regression models were conducted to determine the relative contribution of CSF biomarker measurements to the AD+MCI-AD diagnosis. The first model included hypocretin-1, tau, $A \beta_{42}$, and age (Model 1), and the second included hypocretin-1, P-tau, $\mathrm{A} \beta_{42}$, and age (Model 2) (Table 3 ). After adjusting for these variables, hypocretin-1 levels remained significantly and independently associated with AD+MCI-AD, with an OR of $2.70(95 \% C I=[1.37-5.32])$ for Model 1 and an OR of $2.66(95 \% C I=[1.35-5.23])$ for Model 2, exceeding that for $A \beta_{42}$ (Table 3 ).

\section{CORRELATIONS BETWEEN CSF AD BIOMARKERS AND HYPOCRETIN-1}

We found a positive correlation between hypocretin-1 levels and $\mathrm{A} \beta_{42}(r=0.43, p=0.001)$ and the IATI index $(r=0.42$, $p=0.002)$ in the AD+MCI-AD group $(n=53)$. This can be explained by the patient group with advanced AD (AD: $r=$ $0.45, p=0.005$; MCI due to AD: $r=0.38, p=0.15)$. No significant correlations were found between hypocretin- 1 and other biomarkers in the DEM group. In the whole group of 91

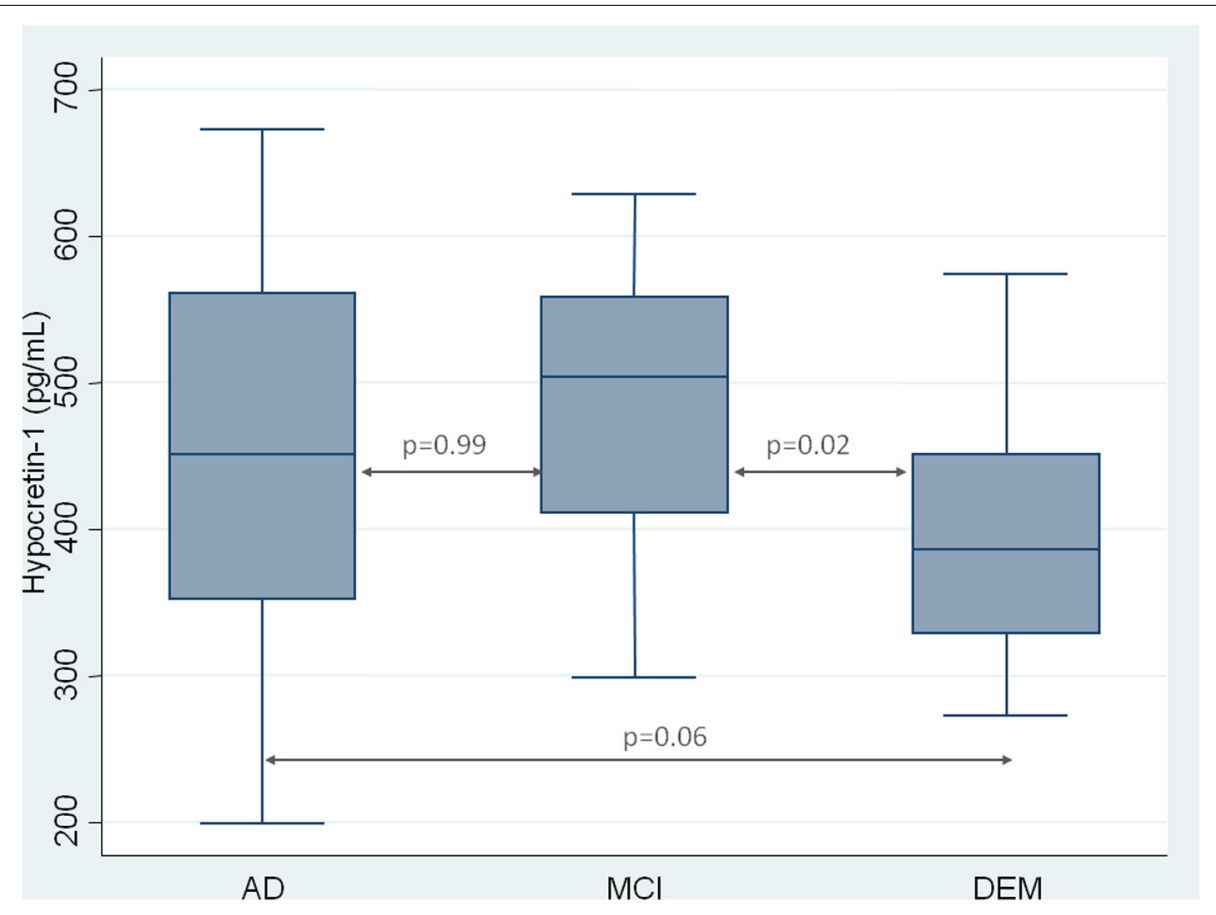

FIGURE 1 | CSF measurement of hypocretin-1 in patients with Alzheimer Disease (AD), Mild Cognitive Impairment (MCI), and with others Dementia (DEM). Results are shown as a box-whisker plot, with median and 25th quartile of CSF hypocretin-1 levels. 
Table 2 | Comparison of sociodemographic, clinical, and biological variables between $A D+M C l$ due to $A D$ patients and patients with other etiology of dementia.

\begin{tabular}{|c|c|c|c|c|c|c|}
\hline & \multicolumn{2}{|c|}{$\begin{array}{c}\text { DEM } \\
N=\mathbf{3 8}\end{array}$} & \multicolumn{2}{|c|}{$\begin{array}{c}A D+M C l \text { due to } A D \\
N=53\end{array}$} & $p$-value & $p$-value ${ }^{a}$ \\
\hline Age (years) & \multicolumn{2}{|c|}{66.84 [32.07-85.15] } & \multicolumn{2}{|c|}{72.58 [49.80-89.49] } & 0.02 & \\
\hline Male & 24 & 63.16 & 27 & 50.94 & \multirow[t]{2}{*}{0.25} & \\
\hline Female & 14 & 36.84 & 26 & 49.06 & & \\
\hline MMSE & \multicolumn{2}{|c|}{20.50 [9.00-30.00] } & & $-30.00]$ & \multicolumn{2}{|l|}{0.09} \\
\hline $\mathrm{A} \beta_{42}(\mathrm{pg} / \mathrm{ml})$ & \multicolumn{2}{|c|}{694.00 [306.00-1645.00] } & \multicolumn{2}{|c|}{568.00 [232.00-1811.00] } & \multirow[t]{2}{*}{0.01} & \multirow[t]{2}{*}{0.03} \\
\hline \multicolumn{5}{|l|}{$\mathrm{A} \beta_{42}(\mathrm{pg} / \mathrm{ml})$} & & \\
\hline$\leq 500$ & 8 & 21.05 & 19 & 35.85 & \multirow[t]{2}{*}{0.13} & \multirow[t]{2}{*}{0.19} \\
\hline$>500$ & 30 & 78.95 & 34 & 64.15 & & \\
\hline Tau (pg/ml) & \multicolumn{2}{|c|}{264.50 [104.00-1200.00] } & \multicolumn{2}{|c|}{588.00 [283.00-2165.00] } & $<0.0001$ & \multirow[t]{2}{*}{$<0.0001$} \\
\hline \multicolumn{6}{|l|}{ Tau (pg/ml) } & \\
\hline \multicolumn{7}{|l|}{ P-tau (pg/ml) } \\
\hline$\geq 60$ & 7 & 18.42 & 46 & 86.79 & \multirow[t]{2}{*}{$<0.0001$} & \multirow[t]{2}{*}{$<0.0001$} \\
\hline$<60$ & 31 & 81.58 & 7 & 13.21 & & \\
\hline IATI index & & $-3.55]$ & & $-2.53]$ & $<0.0001$ & $<0.0001$ \\
\hline \multicolumn{7}{|l|}{ IATI Index } \\
\hline$<1$ & 16 & 43.24 & 47 & 88.68 & \multirow[t]{2}{*}{$<0.0001$} & \multirow[t]{2}{*}{$<0.0001$} \\
\hline$\geq 1$ & 21 & 56.76 & 6 & 11.32 & & \\
\hline Hypocretin-1 (pg/ml) & \multicolumn{2}{|c|}{386.00 [273.00-574.00] } & \multicolumn{2}{|c|}{486.50 [199.00-672.50] } & \multirow[t]{2}{*}{0.004} & 0.005 \\
\hline Hypocretin-1 (pg/ml) & & & & & & \\
\hline 110-200 & 0 & 0.00 & 1 & 1.89 & 0.58 & NA \\
\hline$\geq 200$ & 38 & 100.00 & 52 & 98.11 & & \\
\hline
\end{tabular}

adjustment for age.

${ }^{b} 300 \mathrm{pg} / \mathrm{ml}$ (if age between 21 and 50 years old), 450 (if age between 51 and 70 years old), and 500 (if age > 71 years old).

NA, Not applicable.

Table 3 | Multivariate logistic regression models of CSF biomarker measurements associated with Alzheimer's Disease (AD)/Mild Cognitive impairment due to AD.

\begin{tabular}{|c|c|c|c|}
\hline \multicolumn{2}{|c|}{ Model 1} & \multicolumn{2}{|c|}{ Model 2} \\
\hline OR for 110 -unit increase & $2.70[1.37-5.32]$ & OR for 110 -unit increase & $2.66[1.35-5.23]$ \\
\hline Tau (pg/ml) & & p-Tau (pg/ml) & \\
\hline OR for 300-unit increase & $4.24[2.03-8.84]$ & OR for 60 -unit increase & 12.49 [3.61-43.23] \\
\hline Age (years) & & Age (years) & \\
\hline OR for 10-year increase & $2.15[1.05-4.40]$ & OR for 10 -year increase & 1.94 [0.96-3.92] \\
\hline
\end{tabular}

${ }^{a} A D+M C l$ due to $A D$ vs. DEM.

cognitive patients, the expected negative correlations between $\mathrm{A} \beta_{42}$ and tau $(r=-0.3, p=0.005)$ and between $\mathrm{A} \beta_{42}$ and $\mathrm{P}$ tau $(r=-0.22, p=0.04)$ were found, but not for hypocretin-1 levels. Unexpectedly, we found positive correlations between CSF $\mathrm{A} \beta_{42}$ and tau $(r=0.69, p=0.004)$ and P-tau $(r=0.70, p=$ $0.004)$ in patients with NC, but not for hypocretin-1 levels.

\section{ASSOCIATIONS BETWEEN CSF BIOMARKERS AND SLEEP DISTURBANCES}

Sleep disturbances were assessed by clinical interview and questionnaires in a limited population sample $(n=32)$ with the help of caregivers when required. The mean MMSE for the $20 \mathrm{AD}$ patients who completed sleep questionnaires is 19.9 [14-24], and 
21.6 [14-26] for the 3 DEM patients. No associations were found between sleep disturbances (wake time after sleep onset, total sleep time, and Epworth Sleepiness Scale) and CSF biomarkers $\left(\mathrm{A} \beta_{42}\right.$, tau, P-tau, and hypocretin-1) in either the whole assessed population or in the $\mathrm{AD}+\mathrm{MCI}-\mathrm{AD}$ group only $(n=29)$.

\section{DISCUSSION}

We investigated the relationships between CSF $A \beta_{42}$, tau, P-tau, and hypocretin-1 levels in a large clinically based cohort of patients with different etiologies of cognitive abnormalities and hypocretin-deficient narcoleptic patients. Higher CSF hypocretin-1 levels were found in all demented patients compared to control narcoleptic patients without cognitive abnormalities. More interestingly, CSF hypocretin-1 levels were higher in patients with MCI due to AD compared to patients with other etiologies of dementia, with a similar tendency for advanced AD. Because all patients with MCI in our cohort evolved toward the $\mathrm{AD}$ process, we pooled the $\mathrm{AD}$ patients into an early and advanced stage AD group. Multivariate analysis results suggest that CSF hypocretin-1 concentrations could contribute to the AD diagnosis, as significant levels were found in early stage AD. Moreover, a positive correlation between $A \beta_{42}$ and hypocretin-1 levels was observed in advanced $\mathrm{AD}$, with no association with sleep disturbances measured by sleep questionnaires only. Furthermore, no association was found between CSF, tau, or P-tau and hypocretin1 levels.

CSF biomarkers are widely used for diagnosing $\mathrm{AD}$ in atypical clinical forms and for differential and early diagnosis (Bateman et al., 2007; Blennow and Zetterberg, 2009; Mattsson et al., 2009; Gabelle et al., 2011). A major contribution of our study is that it underscores the greater contribution of using hypocretin-1 compared to $A \beta_{42}$ to differentiate an $A D+M C I$ due to $A D$ diagnosis from other dementia diagnoses. Although our results confirmed that common amyloid and tau CSF biomarkers help diagnose AD even at early stages, more interestingly, hypocretin-1 is significantly and independently associated with this group of patients, with an OR of 2.70 after full adjustment. The diagnostic interest of hypocretin-1 is also highlighted by the fact that its CSF concentrations differed significantly between DEM and MCI patients that converted into AD. This indicates that hypocretin-1 could be used for early detection of AD. The underlying rationale is that amyloid pathology is believed to be present very early in the development of the disease, and $A \beta_{42}$ and hypocretin were correlated in AD. Previous studies have shown normal CSF hypocretin-1 levels in AD (Mignot et al., 2002; Dauvilliers et al., 2003), and normal or low levels in LBD (Baumann et al., 2004; Wennstrom et al., 2012). However, to our knowledge, none of these studies compared patients at different stages of the AD process, and none specifically included patients affected with MCI. Betweengroup comparisons of CSF hypocretin-1 levels in patients with different etiologies of cognitive abnormalities are rare, and pathological cutoffs for CSF hypocretin-1 levels have been validated for narcolepsy only (Mignot et al., 2002; Dauvilliers et al., 2003, 2007). One study reported a $40 \%$ reduction in the total number of hypocretin neurons in AD (Fronczek et al., 2012), but no correlation has yet been reported between CSF hypocretin1 levels and the number of hypocretin neurons in humans, as surviving neurons might compensate for lost neurons by increasing hypocretin synthesis.

Another striking finding of the present study is the positive correlation between hypocretin- 1 and $A \beta_{42}$ levels in $\mathrm{AD}$, but not in other dementias or in NC. Because the patients with MCI were seen regularly at the CMRR, with follow-up revealing conversion to $\mathrm{AD}$, we were able to determine largely pronounced brain $\beta$-amyloid and hypocretin-1 interactions in advanced stage AD. Using in vivo microdialysis in APP transgenic mice, a seminal study highlighted significant interactions between brain extracellular accumulations of $A \beta$ peptide, wakefulness, and hypocretin- 1 (Kang et al., 2009). The same authors recently demonstrated a strong relationship between disrupted sleep-wake states and increased wakefulness, loss of circadian $A \beta$ fluctuation, and neuronal activity (through lactate metabolism) in APP transgenic mice only after amyloid plaque formation, and changes were prevented by $\mathrm{A} \beta_{42}$ active immunization (Roh et al., 2012).

Human studies of CSF samples collected via lumbar catheters in young healthy volunteers have revealed peak CSF A $\beta$ concentrations in the evening and lower concentrations overnight (Bateman et al., 2007; Kang et al., 2009). A slight correlation between $A \beta_{42}$ and hypocretin-1 levels was recently reported in 6 patients with $\mathrm{AD}$ and 6 healthy controls, with no between-group difference in CSF hypocretin-1 levels and its circadian amplitude (Slats et al., 2012). In contrast, no association between $A \beta_{42}$ and hypocretin-1 was reported in either control subjects or patients with $\mathrm{AD}$ in a larger study, but with low hypocretin-1 levels in 9 female patients with LBD (Wennstrom et al., 2012). In young patients with $\mathrm{AD}$ carrying presenilin mutations, CSF $\mathrm{A} \beta$ fluctuation disappeared after amyloid plaque formation (Roh et al., 2012). These varying results on relationships between brain $A \beta$ and hypocretin-1 levels across human studies may be due to small sample sizes, differences in methodology and population characteristics, and the influence of uncontrolled factors such as sleep-wake states.

Sleep-wake behavior is a fundamental brain function that is substantially associated with cognition and synaptic plasticity (Tononi and Cirelli, 2006). A recent study in mice reported that sleep is critical for ensuring metabolic homeostasis: large increases in the cortical interstitial space during sleep result in convective exchange between CSF and the interstitial fluid (Xie et al., 2013). The restorative function of sleep may therefore be related to increased clearance of potentially neurotoxic degradation products of neuronal activity that accumulate in the awake brain, such as A $\beta$ (Xie et al., 2013). Daytime wake and nighttime sleep fragmentation have been frequently reported in $\mathrm{AD}$, but with an unclear biological basis (Montplaisir et al., 1995; Dauvilliers et al., 2003; Kang et al., 2009). Because hypocretin is a major wake-related neurotransmitter (de Lecea et al., 1998), few studies have focused on its impact on wakefulness consolidation in either patients with AD (Friedman et al., 2007) or elderly subjects (Ju et al., 2013; Spira et al., 2013). Using wrist actigraphy recording, one study reported increased wake fragmentation in patients with lower CSF hypocretin-1 levels (Friedman et al., 2007). A recent study revealed an association between CSF A $\beta_{42}$ and poor sleep quality, but not sleep quantity, using actigraphy in cognitively normal subjects potentially in the preclinical stage of 
$\mathrm{AD}$ (i.e., 50\% having parental history of late-onset AD) (Ju et al., 2013). Recently, a self-reported assessment of sleep parameters in community-dwelling older adults showed that both shorter sleep duration and poorer sleep quality were associated with greater $\mathrm{A} \beta$ burden, measured by Pittsburgh compound B positron emission tomography (Spira et al., 2013). Unfortunately, as in the previous study, our protocol did not include actigraphy or polysomnography recordings. To determine the impact of sleep/wake states on the interaction between CSF $A \beta_{42}$ and hypocretin-1 levels, we determined the presence of sleep disturbances in a limited population sample using clinical interviews and questionnaires with patients, with caregiver help when required. We found no associations between total sleep time, wake time after sleep onset, daytime sleepiness, and any CSF biomarkers. Further studies using objective tools to assess sleep disturbances in patients with $\mathrm{AD}$ are recommended as clinical interviews have limited value to assess this condition.

The degeneration of key sleep and wake regulatory systems in $\mathrm{AD}$, which is responsible for a marked increase in nighttime wakefulness and daytime sleepiness, may interfere with neuronal metabolism per se and ultimately lead to $\mathrm{A} \beta$ aggregation. Interestingly in $\mathrm{AD}$, the decrease in CSF $A \beta_{42}$ associated with its aggregation in amyloid plaques is also correlated with other $A \beta$ isoforms, including $A \beta_{40}$ and soluble APPs, which are released mainly during physiological synaptic plasticity (Cole and Vassar, 2008; Gabelle et al., 2010). It is therefore possible that several factors believed to have a trophic function may play a role in this pathophysiology, with potential relationships with sleep-wake states (Friedman et al., 2007; Cole and Vassar, 2008; Xie et al., 2013). Although the process is largely unclear, amyloid plaque formation, a pathological hallmark of $\mathrm{AD}$, may be modulated by the endogenous hypocretin signal.

Another limitation of the present study is the absence of healthy controls. However it was difficult to construct an appropriate control group of cognitively normal elderly adults with CSF lumbar puncture. We used CSF samples from elderly patients with NC characterized by daytime sleepiness, fragmented nighttime sleep, cataplexy, and particularly hypocretin deficiency. As expected, CSF $A \beta_{42}$, tau, P-tau, and IATI levels differed significantly between $\mathrm{AD}, \mathrm{MCI}$ due to $\mathrm{AD}$, and $\mathrm{NC}$ groups. However, patients with NC with low CSF hypocretin-1 levels did not present low $A \beta_{42}$ levels. Therefore, the positive correlation between CSF $A \beta_{42}$ and hypocretin-1 levels appears to be specific to the $\mathrm{AD}$ process. However, our results in $\mathrm{NC}$ contrasted with a previous case report of a hypocretin-deficient young girl with NC with low CSF $A \beta_{42}$ who had been vaccinated for pandemic H1N1 (Kallweit et al., 2012). Unexpectedly, we also found positive correlations between CSF A $\beta_{42}$ and tau and P-tau levels in patients with $\mathrm{NC}$ with no cognitive abnormalities, but no relationship was found with hypocretin-1. These findings shed light on the complex relationship between these biomarkers and various underlying conditions.

To conclude, we examined CSF $\mathrm{A} \beta_{42}$, tau, P-tau, and hypocretin-1 levels in a large clinically based population of patients with cognitive abnormalities. Results indicate that significantly higher CSF hypocretin-1 concentrations in early stage $\mathrm{AD}$ could contribute to the $\mathrm{AD}$ diagnosis. These results also suggest a pathophysiological relationship between a key wakerelated neurotransmitter (hypocretin-1) and brain accumulation of $\beta$-amyloid peptides in the $\mathrm{AD}$ process. Further longitudinal studies are required to validate these biomarker interactions and to determine the cause-effect relationship and the role of wake/sleep behavior in the regulation of amyloid plaque formation.

\section{REFERENCES}

American Academy of Sleep Medicine. (2005). The International Classification of Sleep Disorders, 2nd Edn. Diagnostic and Coding Manual. Westchester, IL: American Academy of Sleep Medicine.

Bateman, R. J., Wen, G., Morris, J. C., and Holtzman, D. M. (2007). Fluctuations of CSF amyloid-beta levels: implications for a diagnostic and therapeutic biomarker. Neurology 68, 666-669. doi: 10.1212/01.wnl.0000256043. 50901.e3

Baumann, C. R., Dauvilliers, Y., Mignot, E., and Bassetti, C. L. (2004). Normal CSF hypocretin-1 (orexin A) levels in dementia with Lewy bodies associated with excessive daytime sleepiness. Eur. Neurol. 52, 73-76. doi: 10.1159/0000 79749

Blennow, K., and Zetterberg, H. (2009). Cerebrospinal fluid biomarkers for Alzheimer's disease. J. Alzheimers Dis. 18, 413-417. doi: 10.3233/JAD-2009-1177

Boeve, B. F., Maraganore, D. M., Parisi, J. E., Ahlskog, J. E., Graff-Radford, N., Caselli, R. J., et al. (1999). Pathologic heterogeneity in clinically diagnosed corticobasal degeneration. Neurology 53, 795-800. doi: 10.1212/WNL.53.4.795

Braak, H., and Braak, E. (1996). Evolution of the neuropathology of Alzheimer's disease. Acta Neurol. Scand. Suppl. 165, 3-12. doi: 10.1111/j.16000404.1996.tb05866.x

Cole, S. L., and Vassar, R. (2008). The role of amyloid precursor protein processing by BACE1, the beta-secretase, in Alzheimer disease pathophysiology. J. Biol. Chem. 283, 29621-29625. doi: 10.1074/jbc.R800015200

Dauvilliers, Y. (2007). Insomnia in patients with neurodegenerative conditions. Sleep. Med. 8(Suppl. 4), S27-S34. doi: 10.1016/S1389-9457(08)70006-6

Dauvilliers, Y., Arnulf, I., and Mignot, E. (2007). Narcolepsy with cataplexy. Lancet 369, 499-511. doi: 10.1016/S0140-6736(07)60237-2

Dauvilliers, Y., Baumann, C. R., Carlander, B., Bischof, M., Blatter, T., Lecendreux, M., et al. (2003). CSF hypocretin-1 levels in narcolepsy, Kleine-Levin syndrome, and other hypersomnias and neurological conditions. J. Neurol. Neurosurg. Psychiatry 74, 1667-1673. doi: 10.1136/jnnp.74.12.1667

de Lecea, L., Kilduff, T. S., Peyron, C., Gao, X., Foye, P. E., Danielson, P. E., et al. (1998). The hypocretins: hypothalamus-specific peptides with neuroexcitatory activity. Proc. Natl. Acad. Sci. U.S.A. 95, 322-327. doi: 10.1073/pnas.95.1.322

Duyckaerts, C., Delatour, B., and Potier, M. C. (2009). Classification and basic pathology of Alzheimer disease. Acta Neuropathol. 118, 5-36. doi: 10.1007/s00401-009-0532-1

Folstein, M. F., Folstein, S. E., and McHugh, P. R. (1975). Mini-mental state. A practical method for grading the cognitive state of patients for the clinician. J. Psychiatr. Res. 12, 189-198. doi: 10.1016/0022-3956(75)90026-6

Friedman, L. F., Zeitzer, J. M., Lin, L., Hoff, D., Mignot, E., Peskind, E. R., et al. (2007). In Alzheimer disease, increased wake fragmentation found in those with lower hypocretin-1. Neurology 68, 793-794. doi: 10.1212/01.wnl.0000256731.57544.f9

Fronczek, R., van Geest, S., Frolich, M., Overeem, S., Roelandse, F. W., Lammers, G. J., et al. (2012). Hypocretin (orexin) loss in Alzheimer's disease. Neurobiol. Aging 33, 1642-1650. doi: 10.1016/j.neurobiolaging.2011.03.014

Gabelle, A., Roche, S., Geny, C., Bennys, K., Labauge, P., Tholance, Y., et al. (2010). Correlations between soluble alpha/beta forms of amyloid precursor protein and Abeta38, 40, and 42 in human cerebrospinal fluid. Brain Res. 1357, 175-183. doi: 10.1016/j.brainres.2010.08.022

Gabelle, A., Roche, S., Geny, C., Bennys, K., Labauge, P., Tholance, Y., et al. (2011). Decreased sAbetaPPbeta, Abeta38, and Abeta40 cerebrospinal fluid levels in frontotemporal dementia. J. Alzheimers Dis. 26, 553-563. doi: 10.3233/JAD2011-110515

Hulstaert, F., Blennow, K., Ivanoiu, A., Schoonderwaldt, H. C., Riemenschneider, M., de Deyn, P. P., et al. (1999). Improved discrimination of AD patients using beta-amyloid(1-42) and tau levels in CSF. Neurology 52, 1555-1562. doi: 10.1212/WNL.52.8.1555 
Ju, Y. E., McLeland, J. S., Toedebusch, C. D., Xiong, C., Fagan, A. M., Duntley, S. P., et al. (2013). Sleep quality and preclinical Alzheimer disease. JAMA Neurol. 70, 587-593. doi: 10.1001/jamaneurol.2013.2334

Kallweit, U., Hidalgo, H., Engel, A., Baumann, C. R., Bassetti, C. L., and Dahmen, N. (2012). Post H1N1 vaccination narcolepsy-cataplexy with decreased CSF beta-amyloid. Sleep. Med. 13, 323. doi: 10.1016/j.sleep.2011.10.023

Kang, J. E., Lim, M. M., Bateman, R. J., Lee, J. J., Smyth, L. P., Cirrito, J. R., et al. (2009). Amyloid-beta dynamics are regulated by orexin and the sleep-wake cycle. Science 326, 1005-1007. doi: 10.1126/science.1180962

Mattsson, N., Zetterberg, H., Hansson, O., Andreasen, N., Parnetti, L., Jonsson, M., et al. (2009). CSF biomarkers and incipient Alzheimer disease in patients with mild cognitive impairment. JAMA 302, 385-393. doi: 10.1001/jama. 2009.1064

McKeith, I. G. (2006). Consensus guidelines for the clinical and pathologic diagnosis of dementia with Lewy bodies (DLB): report of the Consortium on DLB International Workshop. J. Alzheimers Dis. 9, 417-423. doi: 10.1212/WNL.47.5. 1113

McKeith, I. G., Galasko, D., Kosaka, K., Perry, E. K., Dickson, D. W., Hansen, L. A., et al. (1996). Consensus guidelines for the clinical and pathologic diagnosis of dementia with Lewy bodies (DLB): report of the consortium on DLB international workshop. Neurology 47, 1113-1124. doi: 10.1212/WNL.47. 5.1113

McKhann, G., Drachman, D., Folstein, M., Katzman, R., Price, D., and Stadlan, E. M. (1984). Clinical diagnosis of Alzheimer's disease: report of the NINCDSADRDA Work Group under the auspices of Department of Health and Human Services Task Force on Alzheimer's Disease. Neurology 34, 939-944. doi: 10.1212/WNL.34.7.939

McKhann, G. M., Albert, M. S., Grossman, M., Miller, B., Dickson, D., Trojanowski, J. Q., et al. (2001). Clinical and pathological diagnosis of frontotemporal dementia: report of the Work Group on Frontotemporal Dementia and Pick's Disease. Arch. Neurol. 58, 1803-1809. doi: 10.1001/archneur.58.11.1803

McKhann, G. M., Knopman, D. S., Chertkow, H., Hyman, B. T., Jack, C. R. Jr., Kawas, C. H., et al. (2011). The diagnosis of dementia due to Alzheimer's disease: recommendations from the National Institute on Aging-Alzheimer's Association workgroups on diagnostic guidelines for Alzheimer's disease. Alzheimers Dement 7, 263-269. doi: 10.1016/j.jalz.2011.03.005

Mignot, E., Lammers, G. J., Ripley, B., Okun, M., Nevsimalova, S., Overeem, S., et al. (2002). The role of cerebrospinal fluid hypocretin measurement in the diagnosis of narcolepsy and other hypersomnias. Arch. Neurol. 59, 1553-1562. doi: 10.1001/archneur.59.10.1553

Montplaisir, J., Petit, D., Lorrain, D., Gauthier, S., and Nielsen, T. (1995). Sleep in Alzheimer's disease: further considerations on the role of brainstem and forebrain cholinergic populations in sleep-wake mechanisms. Sleep 18, 145-148.
Petersen, R. C., Smith, G. E., Waring, S. C., Ivnik, R. J., Tangalos, E. G., and Kokmen, E. (1999). Mild cognitive impairment: clinical characterization and outcome. Arch. Neurol. 56, 303-308. doi: 10.1001/archneur.56.3.303

Roh, J. H., Huang, Y., Bero, A. W., Kasten, T., Stewart, F. R., Bateman, R. J., et al. (2012). Disruption of the sleep-wake cycle and diurnal fluctuation of beta-amyloid in mice with Alzheimer's disease pathology. Sci. Transl. Med. 4, 150ra122. doi: 10.1126/scitranslmed.3004291

Slats, D., Claassen, J. A., Lammers, G. J., Melis, R. J., Verbeek, M. M., and Overeem, S. (2012). Association between hypocretin-1 and amyloid-beta42 cerebrospinal fluid levels in Alzheimer's disease and healthy controls. Curr. Alzheimer Res. 9, 1119-1125. doi: 10.2174/156720512804142840

Slats, D., Claassen, J. A., Verbeek, M. M., and Overeem, S. (2013). Reciprocal interactions between sleep, circadian rhythms and Alzheimer's disease: focus on the role of hypocretin and melatonin. Ageing Res. Rev. 12, 188-200. doi: 10.1016/j.arr.2012.04.003

Spira, A. P., Gamaldo, A. A., An, Y., Wu, M. N., Simonsick, E. M., Bilgel, M., et al. (2013). Self-reported sleep and beta-amyloid deposition in communitydwelling older adults. JAMA Neurol. 70, 1537-1543. doi: 10.1001/jamaneurol. 2013.4258

Tononi, G., and Cirelli, C. (2006). Sleep function and synaptic homeostasis. Sleep. Med. Rev. 10, 49-62. doi: 10.1016/j.smrv.2005.05.002

Wennstrom, M., Londos, E., Minthon, L., and Nielsen, H. M. (2012). Altered CSF orexin and alpha-synuclein levels in dementia patients. J. Alzheimers Dis. 29, 125-132. doi: 10.3233/JAD-2012-111655

Xie, L., Kang, H., Xu, Q., Chen, M. J., Liao, Y., Thiyagarajan, M., et al. (2013). Sleep drives metabolite clearance from the adult brain. Science 342, 373-377. doi: $10.1126 /$ science. 1241224

Conflict of Interest Statement: The authors declare that the research was conducted in the absence of any commercial or financial relationships that could be construed as a potential conflict of interest.

Received: 30 January 2014; accepted: 27 May 2014; published online: 11 June 2014. Citation: Dauvilliers YA, Lehmann S, Jaussent I and Gabelle A (2014) Hypocretin and brain $\beta$-amyloid peptide interactions in cognitive disorders and narcolepsy. Front. Aging Neurosci. 6:119. doi: 10.3389/fnagi.2014.00119

This article was submitted to the journal Frontiers in Aging Neuroscience.

Copyright (c) 2014 Dauvilliers, Lehmann, Jaussent and Gabelle. This is an openaccess article distributed under the terms of the Creative Commons Attribution License (CC BY). The use, distribution or reproduction in other forums is permitted, provided the original author(s) or licensor are credited and that the original publication in this journal is cited, in accordance with accepted academic practice. No use, distribution or reproduction is permitted which does not comply with these terms. 